\title{
Schizophrenia: The Dark Side of Toxoplasmosis
}

\author{
Shahriar Eftekharian ${ }^{1}{ }^{\circledR}$, Sara Rahmati Roodsari ${ }^{2}$, Zahra Arab-Mazar ${ }^{3}$, Mohsen Rahimi $^{4^{*}(\mathbb{C}}$ \\ ${ }^{1}$ Orthodontist, Private Office, Tehran, Iran \\ ${ }^{2}$ Functional Neourosurgery Research Center, Shohada Tajrish comprehensive neurosurgical center of excellence, Shahid \\ Beheshti University of Medical Sciences, Tehran, Iran \\ ${ }^{3}$ Department of Medical Parasitology and Mycology, School of Public Health, Tehran University of Medical Sciences, \\ Tehran, Iran \\ ${ }^{4}$ Research Center for Prevention of Oral and Dental Diseases, Baqiyatallah University of Medical Sciences, Tehran, Iran
}

*Correspondence to Mohsen Rahimi, Research Center for Prevention of Oral and Dental Diseases, Baqiyatallah University of Medical Sciences, Tehran, Iran. Email: mohsen1rahimi@yahoo.com

Published online March 27, 2021

\section{Dear Editor,}

Toxoplasma gondii is an intracellular parasite that has been associated with several mental disorders. It usually causes an inapparent primary infection. Found worldwide, $T$. gondii is capable of infecting virtually all warm-blooded animals. ${ }^{1-3}$

Schizophrenia is a neurological disorder characterized with long-term and devastating neuropsychological problems, usually presenting in adolescents or young adults. The disease affects almost $1.1 \%$ of the global population including all races and ethnic groups with an equal prevalence in both sexes. ${ }^{1}$ T. gondii seroprevalence is strongly associated with 12-month generalized anxiety disorder but not with other anxiety, depressive, or alcoholrelated disorders. Because of the intracellular nature of some parasites, their treatment and development of new drugs is a major challenge for scientists. ${ }^{4}$ Recently, the use of nanoparticles and nano-scaffolds has suggested for the treatment of parasitic diseases, although limited research has been done in this regard..$^{5-7}$

Toxoplasma gondii modulates the secretion of neurotransmitters (such as dopamine) via producing tyrosine hydroxylase and interacting with astrocyte glial cells. Therefore, this parasite can be related to the pathogenesis of schizophrenia. The association between schizophrenia and toxoplasmosis requires researchers to investigate links between this parasitic infection with other neurological disorders such as paranoia, depression, and brain injuries. A higher probability of schizophrenia has been recorded in those who have antibodies against $T$. gondii in their sera. It has been suggested that $T$. gondii is translocated to target cerebral regions via dendritic cells and macrophages. ${ }^{1}$

The parasite cysts have been identified in astrocytes, indicating possible interactions between $T$. gondii cysts and these cells. In the brain tissue experimentally infected with virulent type I T. gondii, parasites were shown to be localized around neuronal nuclei of astrocytes. Therefore, research evidence suggests that $T$. gondii can play the role of a neuromodulator and infect neuronal cells within the brain. ${ }^{1}$

In fact, $T$. gondii cysts have been detected in various cerebral regions including basal ganglia, olfactory bulbs, hippocampus, thalamus, diencephalon, cortex, as well as in the amygdale, further highlighting the possible role of this parasite in brain damages. ${ }^{1}$ It has been suggested that T. gondii may use genes to augment its survival in its intermediate and definitive hosts. For reaching this goal, these genes may facilitate the translocation of the parasite to the brain to promote required behavioral modifications. ${ }^{1}$

The main aim of using nanoparticles/nano-scaffolds is to use them as a drug delivery system and to release the drug agent to affect the specific site. Recently, the use of particle systems such as nanomedicine has been advocated as a physical tool to improve the pharmacokinetic properties of various types of drug molecules in vivo. ${ }^{5-8}$

Another point supporting a link between schizophrenia and T. gondii is that both of these conditions can modulate the brain at early developmental stages. It has been noted that disorders such as schizophrenia, sclerosis, and Parkinson's disease originate at early phases of the brain development. On the other hand, T. gondii is also able to infect the brain even in the perinatal period. ${ }^{1}$

A link has been proposed between T. gondii infection and the onset and progression of schizophrenia and other bipolar disorders. A study showed that T. gondii can infect the brain by creating cysts within its neurons and by producing tyrosine hydroxylase, an enzyme required for dopamine production. ${ }^{1}$

Dopamine regulates sleep cycle, motivation, attention, mood, and social activities. Also, a link between 
dopamine and schizophrenia is well characterized, and all the drugs used to treat schizophrenia target this neurotransmitter. The levels of some neurotransmitters including dopamine (along with norepinephrine, etc) are affected in the patients infected with T. gondii as well; a process which is also known to be part of schizophrenia pathogenesis. ${ }^{1}$ Dopamine is important in regulating perceptional, behavioral, and other functional properties of the brain. Elevated levels of dopamine are related to the pathogenesis of a variety of neurological disorders such as schizophrenia and mania. For this reason, many drugs used to treat neuronal disorders target dopamine in part of their action. ${ }^{1}$

Multiple studies have been conducted in various populations to divulge the association between schizophrenia and T. gondii infection. Overall, these studies suggest a significant association between schizophrenia and toxoplasmosis, which can be the basis for opening a new path for treating psychiatric and neuronal diseases. ${ }^{1}$

\section{Conflict of Interest}

The authors declare that they have no conflict of interests.

\section{Ethical Statement}

Not applicable.

\section{References}

1. Saraei-Sahnesaraei M, Shamloo F, Jahani Hashemi $H$, Khabbaz F, Alizadeh SA. Relation between Toxoplasma gondii infections and schizophrenia. Iran J Psychiatry Clin Psychol.
2009;15(1):3-9. [Persian].

2. Rahimi M, Mohseni M, Bostan H, Parsipour S, Darabi E, Mohammadzadeh T. The prevalence of intestinal parasites in the patients referred to the laboratories of Baqiyatallah hospital during 2010-2014. J Ardabil Univ Med Sci. 2016;15(4):41422. [Persian].

3. Bitaraf HR, Arab-Mazar Z, Ghanimatdan M, Mohammadi M, Mohseni M, Parsipour S, et al. Seroprevalence of Toxoplasma gondii in military personnel and their families referred to the military hospital in Tehran, Iran. Novelty in Biomedicine. 2017;5(4):152-7. doi: 10.22037/nbm.v5i4.16896.

4. Suvisaari J, Torniainen-Holm M, Lindgren M, Härkänen T, Yolken RH. Toxoplasma gondii infection and common mental disorders in the Finnish general population. J Affect Disord. 2017;223:20-5. doi: 10.1016/j.jad.2017.07.020.

5. Taghipour N, Deravi N, Rahimi M. Chitosan-based scaffolds, suitable structures for wound healing dressing: a short review. Regen Reconstr Restor. 2020;5:e11. doi: 10.22037/rrr. v5i1.31130.

6. Rahimi M, Seyyed Tabaei SJ, Ziai SA, Sadri M. Antileishmanial effects of chitosan-polyethylene oxide nanofibers containing berberine: an applied model for Leishmania wound dressing. Iran J Med Sci. 2020;45(4):286-97. doi: 10.30476/ ijms.2019.45784.

7. Seyyed Tabaei SJ, Rahimi M, Akbaribazm M, Ziai SA, Sadri M, Shahrokhi SR, et al. Chitosan-based nano-scaffolds as antileishmanial wound dressing in BALB/C mice treatment: characterization and design of tissue regeneration. Iran J Basic Med Sci. 2020;23(6):788-99. doi: 10.22038/ ijbms.2020.41361.9770.

8. Rahimi M, Arab-Mazar Z, Roodsari SR. Nanomedicine: The Novel Weapon against Parasitic Infections. Novelty in Biomedicine.;8(4):210-211. doi: 10.22037/nbm.v8i4.30416. 